\title{
A Multi-Band U-Strip and SRR Loaded Slot Antenna with Circular Polarization Characteristics
}

\author{
Princy Maria Paul ${ }^{1 *}$, Krishnamoorthy Kandasamy ${ }^{2}$ and Mohammad S. Sharawi ${ }^{3}$ \\ ${ }^{1,2}$ Electronics and Communication Engineering Department, NITK Suratkal, Karnataka, India \\ ${ }^{3}$ Electrical Engineering Department, Polytechnique Montréal, Montréal, QC H3T 1J4, Canada \\ *corresponding author, E-mail: princy7paul @gmail. com
}

\begin{abstract}
A compact multiband circularly polarized slot antenna is proposed here. An F-shaped microstrip feedline is used to excite the square slot antenna loaded with a U-shaped strip and a split ring resonator (SRR) to generate three circularly polarized bands at $1.5 \mathrm{GHz}, 2.75 \mathrm{GHz}$ and $3.16 \mathrm{GHz}$. A meandered slot is used in the feedline and the U-strip to improve the axial ratio bandwidth (ARBW). The meandered feedline excites the slot to produce resonance at $2.5 \mathrm{GHz}$. This resonance along with that of the F-shaped feed, loaded SRR and U-strip combine to give rise to three circularly polarized bands which can be tuned depending on the feed, SRR and U-strip dimensions. The orientation of the F-shaped feed decides the sense of polarization of the three circularly polarized bands of the proposed antenna. The proposed antenna is fabricated on a substrate of FR4 material with dimensions $50 \times 50 \times 1.56 \mathrm{~mm} 3$. The antenna is prototyped and measured in terms of impedance bandwidth, ARBW, gain and efficiency. The simulated and measured results show reasonably good agreement.
\end{abstract}

\section{Introduction}

Multiband antennas are highly demanded nowadays due to their capability to support multiple applications simultaneously when integrated appropriately in various devices. Slot antennas are a class of antennas that can be easily fabricated and mounted on devices, and also offer a low profile and good bandwidth of operation. Hence, the design of novel multiband slot antennas has been investigated widely in the literature [1-3]. Slot antennas have been modified to generate circularly polarized bands with good ARBW, gain and efficiency values at the different resonant bands. Different methods are used conventionally to obtain these characteristics that involve introducing slits or using stubs in the ground plane, which affect the current distribution to generate orthogonal modes with a 90-degree phase shift and hence, circularly polarized (CP) waves [4-5]. Most of the antennas proposed to offer dual bands of operation but are of large size.

In [6], an arrangement of truncations on a patch and slots are used to generate a tri-band CP response. The antenna geometry had a size of $0.28 \lambda_{g}^{2}$, where $\lambda_{g}$ is the guided wavelength corresponding to the lowest resonance frequency. The impedance bandwidths (BWs) obtained and ARBWs at the three bands covered (i.e. $2.3 \mathrm{GHz}, 3.5 \mathrm{GHz}$, $5.5 \mathrm{GHz}$ ) were $4.4 \%, 3.4 \%, 7.2 \%$ and $0.4 \%, 1.1 \%, 0.7 \%$, respectively. Independent control in the sense of polarization for each of the bands was not possible. CP was achieved in dual and triple bands using annular slots as shown in [7] and [8]. In [8], two non-concentric annular slots were etched on a microwave substrate and fed with an L-shaped series step impedance feed configuration to produce $\mathrm{CP}$ resonance bands at $1.22 \mathrm{GHz}, 1.57 \mathrm{GHz}$, and $2.31 \mathrm{GHz}$. The impedance BWs obtained at the three bands were 9\%, 2.5\% and 27\% and the ARBWs obtained were $4.9 \%, 1.2 \%$ and $4.3 \%$, respectively. The proposed antenna had a size of $0.5 \lambda_{g}^{2}$.

Split ring resonators (SRR) were introduced in [9] and used to miniaturize and provide multi-band operation in [10] - [11]. Complementary SRRs (CSRRs) can also be used to obtain negative permittivity effects and hence provide additional resonant bands. CSRRs were used in various monopole and patch antenna designs to generate novel multiband based CP antennas [12]. However, the etching of a CSRR introduces loss and hence, peak gain and radiation efficiency of the antenna are affected. Other techniques used to design $\mathrm{CP}$ based multiband antennas involve the introduction of Electromagnetic Band Gap (EBG) structures and metasurfaces in the designs, but these structures provide a bigger profile, are difficult to fabricate, and most of them offer very narrow axial ratios [13-14]. The loading of simple SRR structures has been used to obtain $\mathrm{CP}$ bands as shown in [15] which involves introducing connected spiral SRRs to a simple square slot antenna to obtain dual band CP antenna. The antenna offered as ARBW of $3.1 \%$ and $4.2 \%$ and at 3.1 $\mathrm{GHz}$ and $4.7 \mathrm{GHz}$, respectively. In [16], a triband $\mathrm{CP}$ slot antenna with ARBWs of $4.37 \%, 11.9 \%$ and $3.6 \%$ at the resonant frequencies was designed using inclined copper strips and SRR loading. The loading of multiple copper strips leads to relatively low efficiency, larger size and narrow ARBW values compared to this proposed work.

In this work, a novel multiband CP square slot antenna is proposed with improved ARBW and efficiency values. The antenna is fed with an F-shaped microstrip line, and loaded with an SRR and U-strip to produce triband operation with centre frequencies at $1.5 \mathrm{GHz}, 2.5 \mathrm{GHz}$, and $3.2 \mathrm{GHz}$. Impedance BWs of $12 \%, 9.1 \%, 7.3 \%$, ARBWs of $9.3 \%, 7.3 \%$, $8.5 \%$ and gains of $3.2 \mathrm{~dB}, 4.5 \mathrm{~dB}$, and $3.5 \mathrm{~dB}$ are obtained at 
the three resonant frequencies, respectively. The proposed antenna is designed for frequencies commonly used for devices pertaining to WiMAX, LTE, Bluetooth and WLAN applications (but with $\mathrm{CP}$ capabilities), but the resonances can be shifted as per requirement depending on SRR, feed and strip dimensions. The orientation of the F-shaped feed line is used to control the sense of polarization of the antenna at the operating bands.

Thus, the proposed antenna offers miniaturized size with improved impedance and AR bandwidth values compared to earlier designs found in the literature. It differs from [16] in that, the proposed antenna uses the resonance frequencies of the SRR and loaded strip along with the effect of the meandered gaps to produce the desired tri-band frequency response. The feed is modified in order to obtain the required input impedance matching and hence improve the S11 and AR responses. Also, the capacitive and inductive effects introduced by the meandered gaps are used to optimize the performance of the antenna. Thus, the antenna can be seen to exhibit superior features using a novel combination of different techniques that involve suitable arrangement and design of the SRR, strips and feed configurations.

\section{Antenna Design and Principle of Operation}

\subsection{Antenna Geometry}

The proposed antenna geometry offering circularly polarized triple band operation is shown in Fig. 1. The antenna is designed on an FR4 substrate of size $50^{\times} 50 \mathrm{~mm}^{2}$, permittivity $(\varepsilon r)=3.6$, height $(h)=1.56 \mathrm{~mm}$ and loss tangent $=0.02$. The top view of the antenna is shown in Fig. 1(a). A square slot of side length (L) of $33.2 \mathrm{~mm}$ is etched out on top of the substrate so that the slot resonates at $2.5 \mathrm{GHz}$. A U-shaped metallic strip is left in the slot. The bottom view of the antenna is shown in Fig. 1(b). A $50 \mathrm{ohm}$ feedline offset from the $\mathrm{x}$-axis by $1.2 \mathrm{~mm}$ is printed at the bottom of the substrate. Two horizontal extension strips are attached to the top of the feedline to make an F-shaped feed. Meandered slots are etched on the right arm of the U-strip and the feedline. Also, an SRR is printed on the bottom layer as in Fig.1 (b) with its dimensions as shown in Fig.1 (c).

\subsection{Operating Principle}

The F-shaped meandered feedline excites the slot so that it produces resonance at its fundamental resonant mode [17]. The magnetic and electric field lines produced upon the excitation of the slot cause the U-strip and the SRR to resonate at their resonant frequencies. The SRR is placed and oriented in the slot such that it can allow for maximum passage of the time-varying axial magnetic field lines through it [18-19]. The SRR structure acts as an LC resonator and can be modelled using the method given in [19]. Here, the SRR dimensions are carefully chosen in order to produce the fundamental resonance at $1.6 \mathrm{GHz}$ upon field excitation. A higher order mode at $2.89 \mathrm{GHz}$ is also observed. The current distributions around the SRR and the inner ring of the SRR can be seen at the two resonance frequencies in Fig. 2(a) and Fig. 2(b) respectively. The maximum current distribution is observed around the inner ring for the higher resonance mode.
The U-strip can resonate at different frequencies depending on its arm lengths.

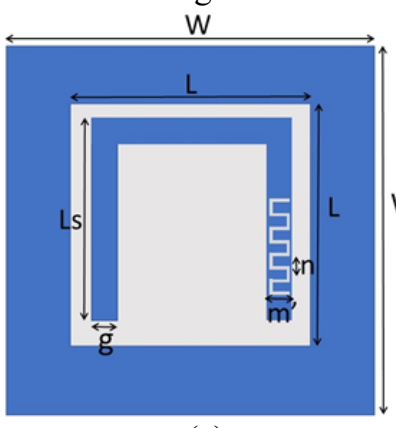

(a)

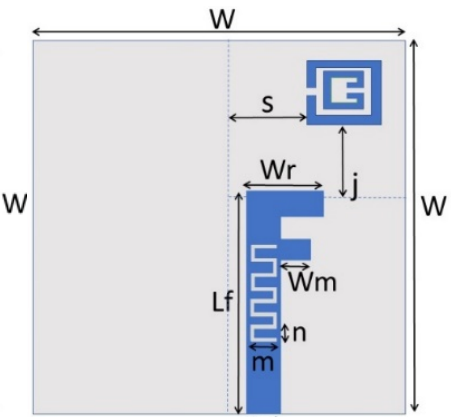

(b)

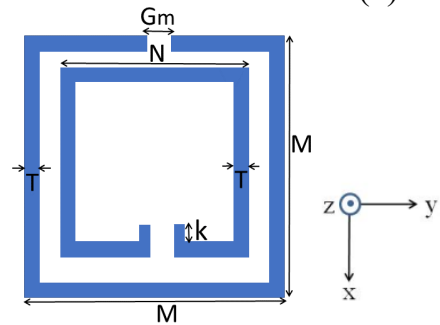

(c)

Figure 1: Geometry of the proposed multiband antenna (a) top view (b) side view (c) SRR structure. $[\mathrm{L}=33.2, \mathrm{~W}=50$, $\mathrm{Wr}=9.6, \mathrm{Wm}=5.3, \mathrm{Lr}=18, \mathrm{Lm}=25, \mathrm{Lf}=31.2, \mathrm{~g}=4, \mathrm{r}=2$, $\mathrm{Wf}=3.6, \mathrm{~s}=8.6, \mathrm{j}=8.4, \mathrm{k}=1.65, \mathrm{M}=6, \mathrm{~N}=4, \mathrm{~T}=0.6, \mathrm{Gm}=0.6$, $\mathrm{h}=1.56, \mathrm{~m}=3, \mathrm{n}=1.4, \mathrm{~m}^{\prime}=3.6$, width of meander $\left.=0.2\right]$, All in $\mathrm{mm}$.

Combination of these with that of the slot and SRR gives rise to the circularly polarized bands of the proposed multiband antenna. The SRR contributes to the lowest resonance frequency hence leading to miniaturization of the proposed design.

The design evolution and the effects of various parts of the antenna are shown in Fig.3. The slot initially resonates at $2.5 \mathrm{GHz}$ when excited with the microstrip feed line. When loading the slot with the U-strip, the resonance is found to shift to $2 \mathrm{GHz}$. Also, a slight resonance is observed around $2.9 \mathrm{GHz}$ which is introduced by the U-strip. The use of the Fshaped feed line lowers the AR value at $2.9 \mathrm{GHz}$. The lowering of AR value could be attributed to the $\mathrm{CP}$ wave obtained upon the combination of the resonances produced by the F-shaped feed and that of the U-strip. The offset at the feedline further improves the AR bandwidth. Also, the first resonance is now slightly shifted to $1.8 \mathrm{GHz}$. Thus, the modified feedline is used to improve the reflection coefficient and AR response of the initial dual-band antenna obtained (without SRR loading). The horizontal stubs of the F shaped feedline and the offset introduce the necessary phase shift required for the orthogonal components that help improve the ARBW values. Besides, the addition of the y-directed horizontal stubs changes the input impedance to the extent that the $\left|S_{11}\right|$ response of the antenna is improved. The improvement in input reflection coefficient characteristics is shown in Fig. 3. Now, loading the structure with an SRR and introducing the meandered slots, additional bands are produced at $2.7 \mathrm{GHz}$ and $3.1 \mathrm{GHz}$. 


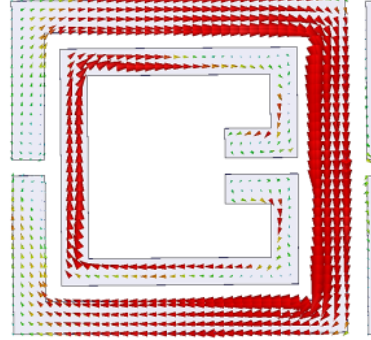

(a)

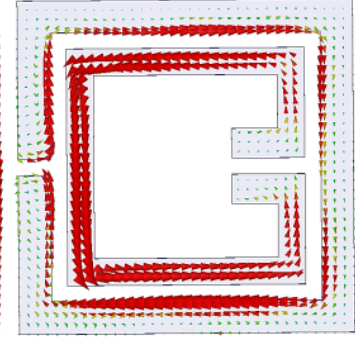

(b)
Figure 2: Surface current distribution for the SRR at (a)1.6 $\mathrm{GHz}$ and (b)2.89 GHz

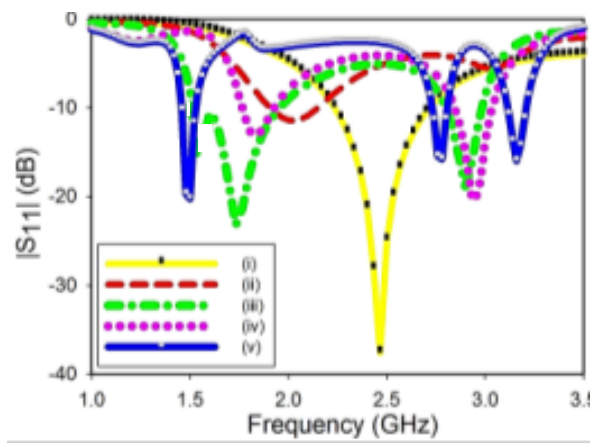

(a)

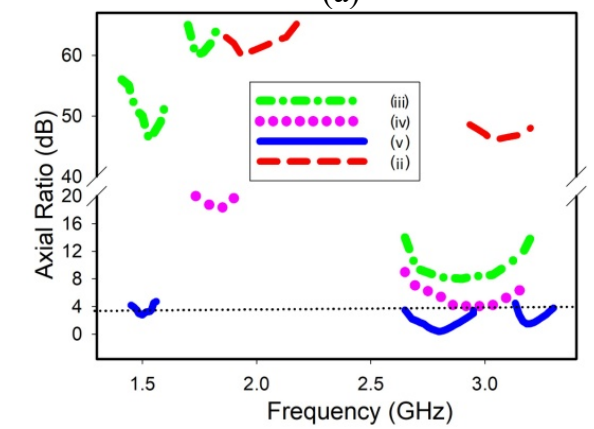

(b)

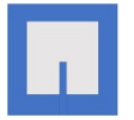

(i)

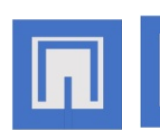

(ii)

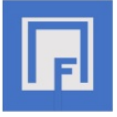

(iii)

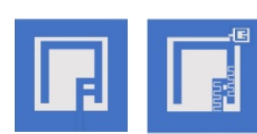

(iv) (v)
Figure 3: Design evolution of proposed antenna and its effects on the simulated:

(a) reflection coefficient curves and (b) axial ratio values with frequency.

Also, the reflection coefficient values at $1.5 \mathrm{GHz}$ are improved and the resonance at $2.9 \mathrm{GHz}$ is shifted to $3.16 \mathrm{GHz}$ due to the fundamental and higher order resonance modes of the SRR [20]. The fundamental resonance produces the CP resonance band at $1.5 \mathrm{GHz}$. The higher order mode of the SRR helps provide the $\mathrm{CP}$ resonance at $2.75 \mathrm{GHz}$. Thus, the resonance bands can be tuned depending on the SRR, Fshaped feedline and U-strip dimensions. The effect of the meandered slots on the AR values at the resonance bands is shown in Fig. 4. The introduction of meandered slots in the feedline and U-strip lower the AR values at the first and third bands, respectively.

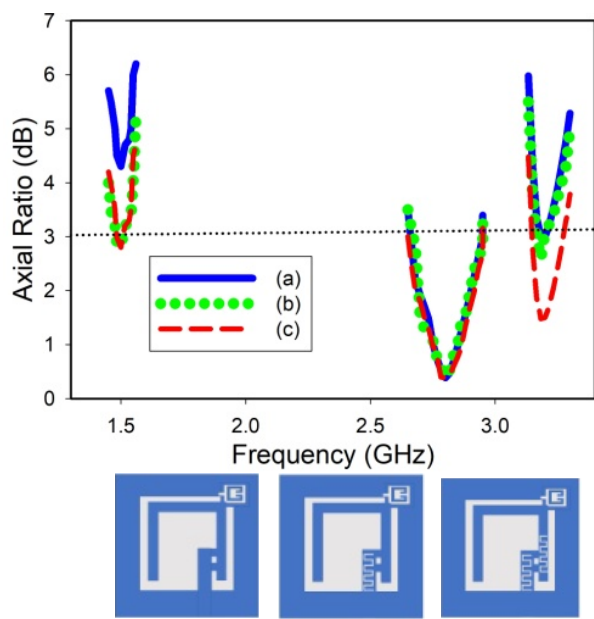

(a)

(b)

(c)

Figure 4: The effect of meandered slots on the simulated axial ratio values for the proposed antenna

\subsubsection{Surface Current Distribution}

In order to understand the $\mathrm{CP}$ operation of the proposed multi-band antenna, current distributions are simulated and studied at each of the three resonance frequencies. The antenna is placed on the $x-y$ plane and the current density vectors are analyzed in the $+z$ direction for phases $\omega \mathrm{t}=0^{\circ}$ and $\omega \mathrm{t}=90^{\circ}$ as shown in Fig. 5. The distributions at the resonant frequencies $1.5 \mathrm{GHz}, 2.75 \mathrm{GHz}$ and $3.16 \mathrm{GHz}$ for the different phases are shown in Fig. 5(a), Fig. 5(b) and Fig. 5(c) respectively, where the dominant currents have been highlighted in each case. The sense of polarization at the three resonance bands is decided by observing the direction of rotation of the current distribution vector from the $+z$ direction. In this case, it is observed that at the resonant frequencies, the vectors rotate in the clockwise direction when there occurs an orthogonal shift in phase. The clockwise direction indicates the presence of right hand circularly polarized (RHCP) waves. The figure clearly shows that the $\mathrm{CP}$ resonance bands at the three resonant frequencies are obtained by exciting the SRR modes (fundamental mode) and U-strip excited by the F-shaped feed, the inner ring of the SRR (higher order mode) and U-strip, and the F-shaped feed and the U-strip. The different lengths of the U-strip when excited, produces the necessary resonance to combine with the other modes to generate the CP bands. In addition, on altering the orientation of the F-shaped feed and the SRR by $90^{\circ}$ and $180^{\circ}$ respectively, reversal of the current direction occurs, hence giving rise to left hand circularly polarized (LHCP) waves at the resonant frequencies. 

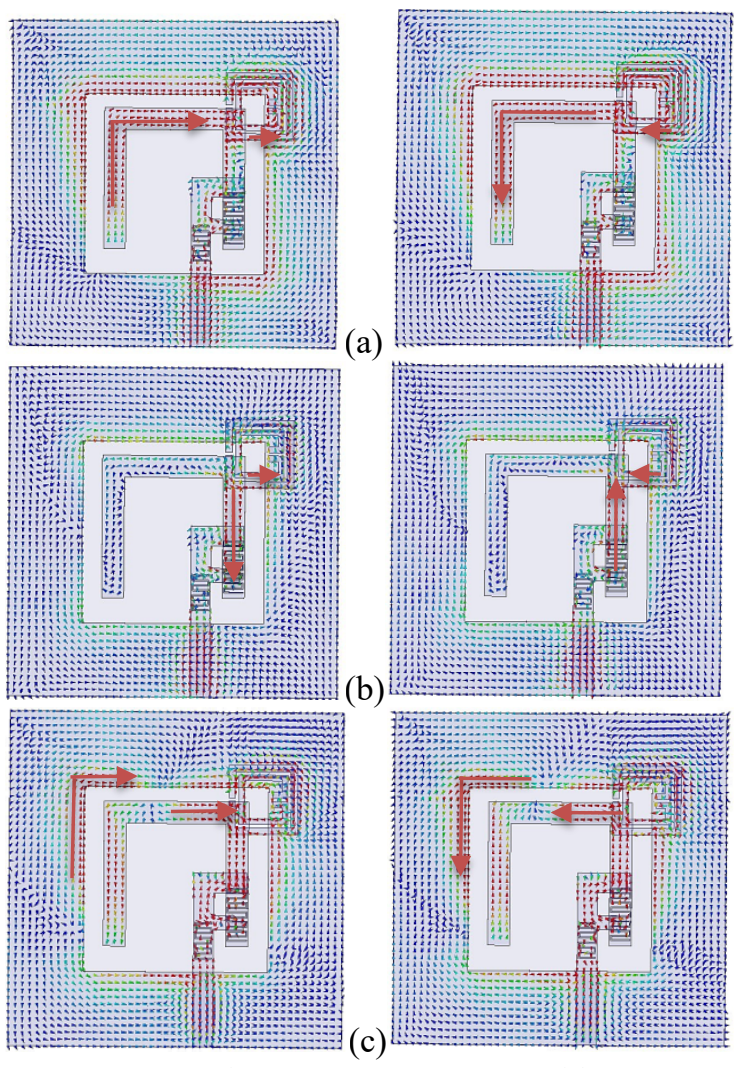

$\omega t=0^{\circ}$

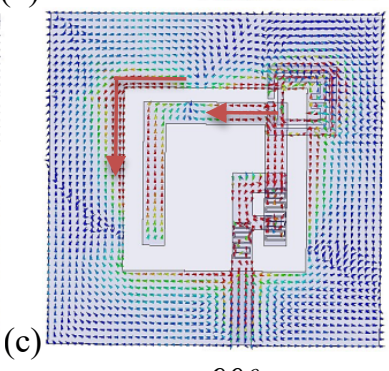

$\omega t=90^{\circ}$

Figure 5: Simulated surface current distribution for the proposed antenna at:

(a) $1.5 \mathrm{GHz}$, (b) $2.75 \mathrm{GHz}$, (c) $3.16 \mathrm{GHz}$

\subsection{2. $\quad$ CP Mechanism}

The CP mechanism is further analyzed by studying the frequency response at each band of operation. The U-strip can be thought of as resonating at different frequencies depending on the different lengths of the U-strip that are excited. The selection of the strip length is done based on the guided wavelengths for different frequencies. The guided wavelength $\left(\lambda_{g}\right)$ for a resonance frequency of $f_{r, G H z}$ is given by [17]:

$$
\lambda_{g}=\frac{300}{f_{r, G H z} \sqrt{\epsilon_{e f f}}}
$$

where effective dielectric constant $\varepsilon_{e f f}$ is calculated as follows:

$$
\epsilon_{e f f}=\frac{\epsilon_{r}+1}{2}+\frac{\epsilon_{r}-1}{2 \sqrt{1+12(h / W f)}}
$$

$W_{f}$ and $h$ denote width of the feedline and thickness of the substrate. The lengths of the strip for different resonance frequencies is taken to be approximately equal to $1 / 2 \lambda_{g}$ at the corresponding frequencies.

The first CP response at $1.5 \mathrm{GHz}$ is found to be obtained due to the fundamental resonance mode of the SRR at 1.6 $\mathrm{GHz}$ and the resonance obtained around $1.4 \mathrm{GHz}$ is due to the excitation of the two arms (L-shape-52mm) of the U-shaped strip. The maximum surface current distribution can be seen around these regions for the first resonance band as shown in Fig 5(a). The meanders at the feed line help to lower the AR values at this band.

The second CP band can be attributed to the higher order resonance mode of the SRR at $2.89 \mathrm{GHz}$ and the right side of the U-strip of $26 \mathrm{~mm}$ which gives a $1 / 2 \lambda_{\mathrm{g}}$ resonance at around $2.65 \mathrm{GHz}$. Thus, a mid-band $\mathrm{CP}$ resonance at $2.75 \mathrm{GHz}$ is obtained. The surface current distribution for this frequency can be seen in Fig. 5(b).

The third CP band arises due to the slot fundamental resonance at $2.5 \mathrm{GHz}$ and the resonance provided by the part of the strip of length $24 \mathrm{~mm}$ that corresponds to a $\lambda_{g}$ of 47.6 $\mathrm{mm}$ (It can be seen from the current distribution that the vectors cancel along the other regions of the U-strip). This value corresponds to a frequency of $3 \mathrm{GHz}$ and hence, a midband resonance at around $2.8 \mathrm{GHz}$ which shifts to $3.17 \mathrm{GHz}$ due to the variation in current and field distributions upon the introduction of the SRR.

\subsubsection{Effect of the meandered gaps}

The effect of the meandered gaps on the frequency response of the proposed antenna is studied based on the design of the gaps and the capacitive effects produced due to them. Consider a set of meandered gaps where $C g$ represents the capacitance of one set of fingers to the ground and $\mathrm{Cm}$ the capacitance between two sets of fingers. Then for an array of $N$ fingers having length $l$, the following equations can be defined:

$$
\begin{gathered}
C_{g}=N C_{1} l / 2 \\
C_{m}=N C_{2} l / 2 \\
\text { Where } C_{1}=C_{e} \quad \begin{array}{c} 
\\
C_{2}=1 /\left(2\left(C_{o}-C_{e}\right)\right)
\end{array}
\end{gathered}
$$

Here $C_{o}$ and $C_{e}$ are defined as the odd mode and even mode capacitances respectively. They can be calculated depending on the dielectric characteristics, the characteristic impedance and phase velocity. It also depends on the finger width and finger separation [21].

The frequency shift due to the effect of the meandered gaps on a strip of length $l o$ can be relatively calculated as [22]:

$$
f_{r, \text { new }}=\sqrt{\frac{l o}{l(N-1)}} f_{r}
$$

Where $f_{r, \text { new }}$ is the new resonance frequency obtained after loading of the meandered gaps. In our proposed design, the fundamental resonance frequency of the slot which depends on the length of the slot $L_{\text {slot }}$ and the dielectric constant of the substrate $\epsilon_{r}$ is calculated as [17]:

$$
f_{r, s l o t}=\frac{c}{2 L_{s l o t}} \sqrt{\frac{2}{1+\epsilon_{r}}}
$$

Here $c$ is the speed of light in vacuum. 
Upon the introduction of the meandered gaps in the feed line, the resonance frequency of the slot is found to shift to $1.59 \mathrm{GHz}$. Thus, lower AR values are observed at the first resonance frequency of $1.5 \mathrm{GHz}$ when the gaps are introduced in the feed line.

The lower half of the U-strip of length $13 \mathrm{~mm}$ resonates at a half wavelength of the frequency $6 \mathrm{GHz}$ which shifts to 3.08 when introducing the gaps. This frequency shift helps lower the AR values at the third band of resonance where the centre frequency obtained is around $3.17 \mathrm{GHz}$.

\subsubsection{Equivalent Circuit Analysis}

The equivalent circuit analysis is done using the circuit models for each electromagnetically coupled component of the design and extracting the corresponding circuit parameters. The equivalent circuit for the unloaded slot is shown in Fig. 6(a) where Ls and Lsh/Csh denote the series inductance of the lossless feedline and the equivalent inductive/capacitive effects of the slot. The equivalent circuit for a set of meandered gaps is shown in Fig. 6(b) where $\mathrm{Ct}$ corresponds to the capacitance of the strips. The total equivalent circuit for the proposed geometry is obtained after including the effect of the meandered gaps as well as that of the mutually coupled SRR which is denoted by a parallel L$\mathrm{C}$ circuit (Le and $\mathrm{Ce}$ ). The complete circuit is shown in Fig. 6(c). The magnetic coupling of the SRR with the slot is characterized by the coupling coefficient $\mathrm{M}$. The series feed inductance of the offset F-shaped feed is denoted as Ls' and that of the U-strip is defined as Lu. The different mutual capacitance effects and gap capacitances introduced by the meandered gaps on the feed and U-strip are denoted as $\mathrm{Cm} / \mathrm{Cg}$ ' and $\mathrm{Cm} / \mathrm{Cg}$ respectively.

The circuit can be simplified as shown in Fig. 6(d) using the equivalent circuit parameters denoted by Ce', Le' and Ce', The parts A and B of the equivalent circuit in Fig. 6(c), show the effect of meandering on the simplified circuit. Upon extraction of the circuit parameters, the resonances for parts $\mathrm{A}$ and $\mathrm{B}$ are obtained to be around $1.5 \mathrm{GHz}$ and $3.15 \mathrm{GHz}$ which explains why the AR values are modified for these resonances due to the additional capacitive effects introduced by the meandering. The equivalent circuit is verified using ADS circuit analysis and the frequency response is plotted. The reflection coefficient values obtained using the circuit analysis and the HFSS simulation are plotted and compared and shown below in Fig. 6(e). The circuit model is found to agree with the results obtained using EM simulation.

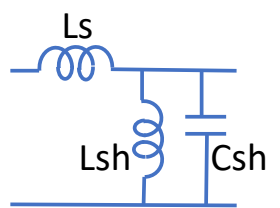

(a)

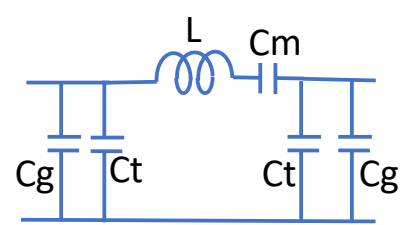

(b)

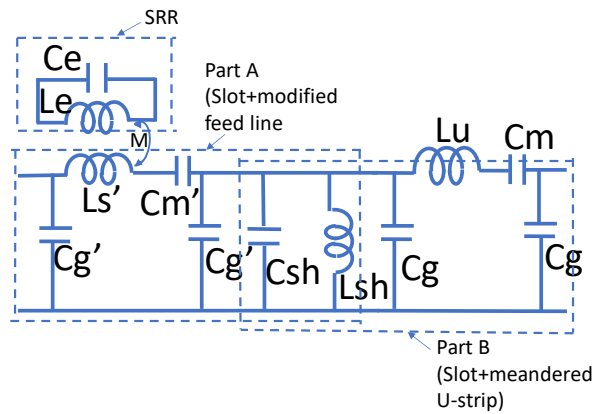

(c)

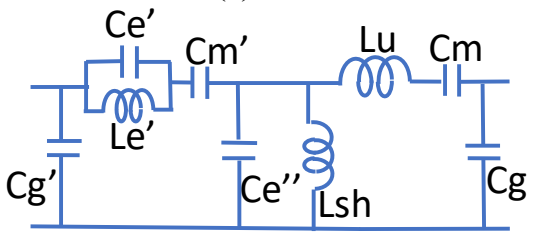

(d)

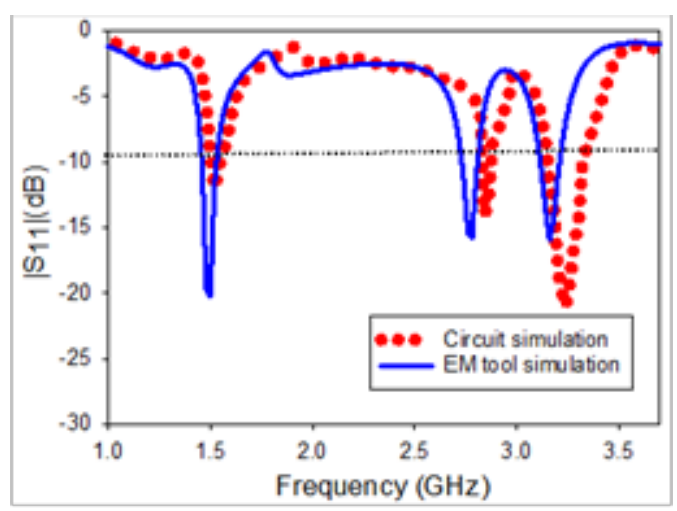

(e)

Figure 6: (a) Equivalent circuit of the unloaded slot with microstrip feed (b) Equivalent circuit model for interdigitated capacitors (c) Equivalent circuit of the proposed antenna (loaded with modified meandered feed, meandered U-strip and loaded SRR) (d) Simplified equivalent circuit for the proposed antenna (e) Reflection coefficient magnitude values for proposed antenna obtained using circuit and EM simulations

\section{Results and Analysis}

The proposed antenna was simulated, fabricated and measured for the reflection coefficient magnitudes and =radiation patterns at the different resonant frequencies. For simulation, the ANSYS HFSS 16.0 software was used. Fabrication and measurement were done at KFUPM using an S103 LPKF machine and an Agilent N9918A Vector Network Analyzer. The top and bottom views of the proposed antenna prototype are shown in Fig. 7. The effects on the simulated and measured reflection coefficient magnitudes and axial ratio values with the change in frequency are plotted as shown in Fig. 8(a) and Fig. 8(b). The simulated and measured results can be observed to be in fairly good agreement. 
The $-10 \mathrm{~dB}$ impedance bandwidths obtained on measurement of the reflection coefficient magnitudes at the resonant frequencies were $10 \%(1.37 \mathrm{GHz}-1.52 \mathrm{GHz}$ with centre frequency of $1.5 \mathrm{GHz}), 9.1 \%(2.62 \mathrm{GHz}-2.87 \mathrm{GHz}$ with centre frequency at $2.75 \mathrm{GHz})$ and $7.3 \%(3.06 \mathrm{GHz}-$ $3.29 \mathrm{GHz}$ with centre frequency at $3.14 \mathrm{GHz}$ ), while the measured ARBWs were 9.3\% $(1.37 \mathrm{GHz}-1.51 \mathrm{GHz}$ with centre frequency at $1.5 \mathrm{GHz}), 7.3 \%(2.65 \mathrm{GHz}-2.85 \mathrm{GHz}$ with centre frequency at $2.75 \mathrm{GHz})$ and $8.5 \%(3.1 \mathrm{GHz}-3.37$ $\mathrm{GHz}$ with centre frequency at $3.15 \mathrm{GHz}$ ).

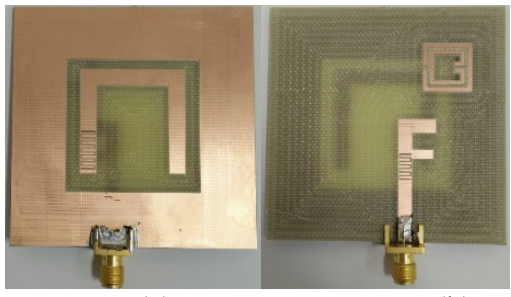

(a)

(b)

Figure 7: Fabricated prototypes

(a) top, and (b) bottom views

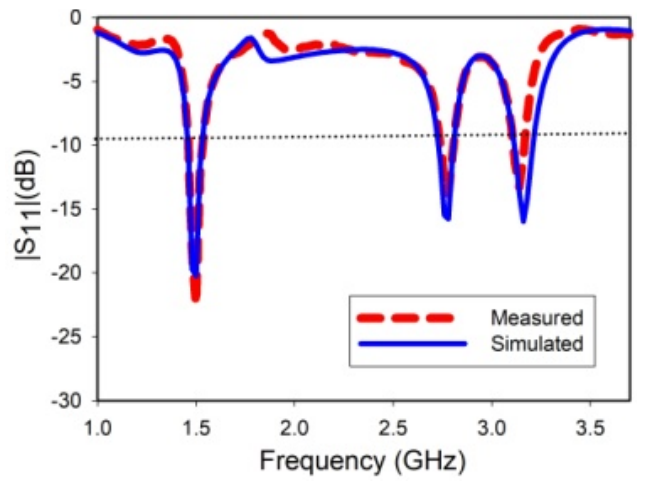

(a)

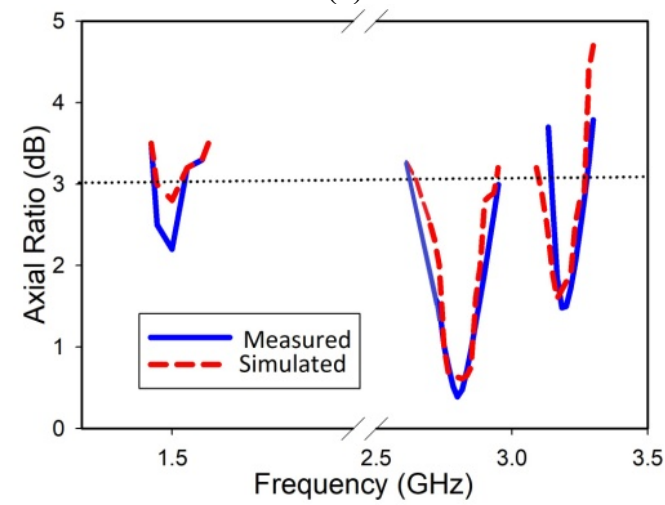

(b)

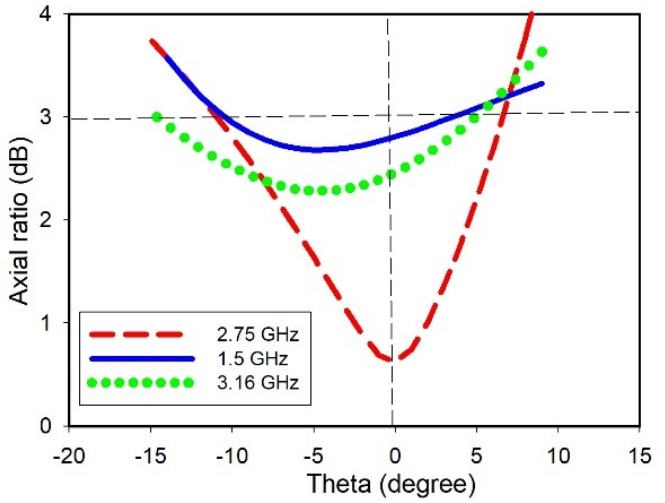

(c)

Figure 8: Variation in characteristics of the proposed antenna (a) reflection coefficient magnitudes, and (b) axial ratio values, versus frequency (c) axial ratio values, versus theta

The variation in the AR values with the elevation angle $(\theta)$ is plotted and shown for the three resonance frequencies in Fig. 8(c). The AR values can be observed to be below $3 \mathrm{~dB}$ for a minimum variation of $+/-5^{\circ}$ from the central axis for the three resonance frequencies. The radiation patterns for the proposed antenna at its resonance frequencies were measured in a Satimo Star-Lab near-field chamber. The values obtained were plotted and compared against the simulated ones for phi $=0^{\circ}$ and phi $=90^{\circ}$ orthogonal planes as seen in Fig. 9. The cross-polarization values are below $-20 \mathrm{~dB}$. In addition, RHCP is radiated in the upper hemisphere. The radiation efficiency and peak gain values obtained on measurement were recorded at the three resonance frequencies as $80 \% / 3.2$ $\mathrm{dB}, 94 \% / 4.5 \mathrm{~dB}$, and $85 \% / 3.5 \mathrm{~dB}$, respectively. The plot seen in Fig. 10 shows the variation of the measured gain and efficiency values with frequency. The proposed design is compared with other multiband $\mathrm{CP}$ slot antenna designs and the various performance parameters are shown in Table 1. Compactness, ease of control in sense of polarization, high impedance BW and ARBW values and good efficiency values are the favourable features in the proposed design compared to other designs in literature.
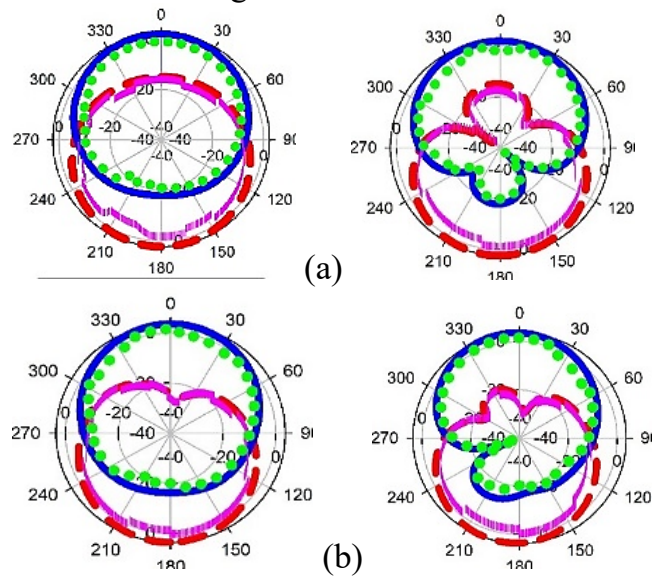

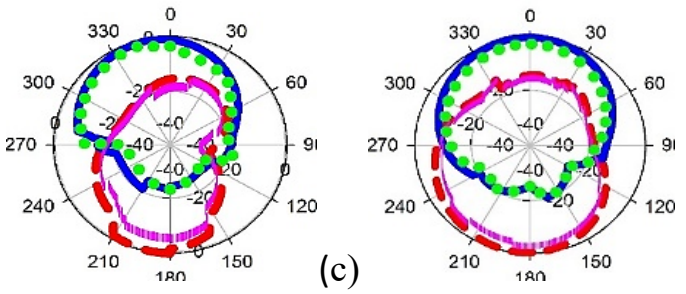

$\mathrm{phi}=0^{\circ}$

phi $=90^{\circ}$

Figure 9.: Radiation patterns obtained for the two orthogonal planes on simulation and measurement of the proposed antenna at: (a) $1.5 \mathrm{GHz}$, (b) $2.75 \mathrm{GHz}$, (c) $3.16 \mathrm{GHz}$ (--LHCP RHCP ||LHCP(measured) ....RHCP (measured) )

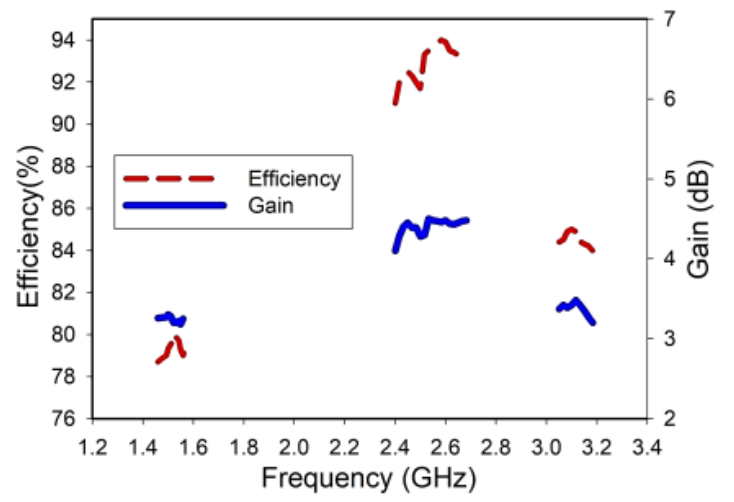

Figure 10: Measured peak gain and radiation efficiency values versus frequency for the proposed antenna.

\section{Conclusions}

Here a compact multiband slot antenna is proposed which operates at three circularly polarized bands and offers wide impedance bandwidth and ARBW. The frequencies of resonance obtained are $1.5 \mathrm{GHz}, 2.75 \mathrm{GHz}$ and $3.16 \mathrm{GHz}$ with measured impedance bandwidths of $10 \%, 9.1 \%$ and $7.3 \%$ and measured ARBWs of $9.3 \%, 7.3 \%$ and $8.5 \%$ at the three resonant bands. A peak gain value of more than $3 \mathrm{dBi}$ and a efficiency higher than $80 \%$ is obtained for the proposed antenna at all the operating bands. The resonant frequency bands can be controlled depending on the feed, U-strip and SRR dimensions, while the polarization sense at the resonant frequencies is controlled upon altering the direction of the Fshaped feed and the SRR.

\section{References}

[1] Cao, Y. F., S. W. Cheung, and T. I. Yuk, A multiband slot antenna for GPS/WiMAX/WLAN systems, IEEE Transactions on Antennas and Propagation, 63 (3), pp. 952-958, 2015

[2] $\mathrm{Hu}, \mathrm{Wei}$, et al., Compact triband square-slot antenna with symmetrical L-strips for WLAN/WiMAX applications, IEEE Antennas and Wireless Propagation Letters, 10, pp. 462-465, 2011.
[3] X.-Q. Zhang, Y.-C. Jiao, and W.-H. Wang, Compact wide tri-band slot antenna for WLAN/WiMAX applications, Electron. Lett., 48 (2), pp. 64-65, 2012.

[4] X. L. Bao and M. J. Ammann, Printed triple-band circularly polarised antenna for wireless systems, Electron. Lett., 50 (23), pp. 1664-1665, 2014.

[5] J. Gyun Baek and K. Cheol Hwang, Triple-band unidirectional circularly polarized hexagonal slot antenna with multiple 1-shaped slits, IEEE Trans. Antennas Propag., 61 (9), pp.4831-4835, 2013.

[6] Xu, Rui, et al., A Design of Triple-Wideband Triple-Sense Circularly Polarized Square Slot Antenna, IEEE Antennas and Wireless Propagation Letters , 2017.

[7] W. Hsieh, T. Chang, and J. Kiang, Dual-band circularly polarized cavity-backed annular slot antenna for GPS receiver, IEEE Trans. Antennas Propag., 60 (4), pp. 2076-2080, 2012.

[8] L.Wang, Y. X. Guo, andW. Sheng, Tri-band circularly polarized annular slot antenna for GPS and CNSS applications, Journal of Electromagnetic Waves and Applications, 26 (14), pp. 1820-1827, 2012.

[9] R. A. Shelby, D. R. Smith, and S. Schultz, Experimental verification of a negative index of refraction, Nature Science, 292 (5514), pp. 77-79, 2011.

[10] P. Paul, K. Kandasamy, M. Sharawi, A Triband Slot Antenna loaded with split-ring resonators, Microwave and Optical Technology Letters, 59 (10), pp. 2638-2645, 2017.

[11] M. U. Khan, and M. S. Sharawi, A $2 \times 1$ multiband MIMO antenna system consisting of miniaturized patch elements, Microwave and Optical Technology Letters, 56 (6), pp.1371-1375, 2014.

[12] L. Zhou, S. Liu, Y. Wei, Y. Chen, and N. Gao, Dualband circularly-polarised antenna based on complementary two turns spiral resonator, Electron. Lett, 46(14), pp.970-971, 2010.

[13] S.-T. Ko, B.-C. Park, and J.-H. Lee, Dual-band circularly polarized patch antenna with first positive and negative modes, IEEE Antennas Wireless Propag. Lett., 12, pp.1165-1168, 2013.

[14] T. V. Hoang, T. T. Le, Q. Y. Li, and H. C. Park, Quadband circularly polarized antenna for $2.4 / 5.3 / 5.8-\mathrm{GHz}$ WLAN and $3.5-\mathrm{GHz}$ WiMAX applications, IEEE Antennas Wireless Propag. Lett., 15, pp.1032-1035, 2016.

[15] K. Kandasamy, B. Majumder, J. Mukherjee and K.P. Ray, Dual-Band Circularly Polarized Split Ring Resonators Loaded Square Slot Antenna, IEEE Trans. Antennas Propag., 64 (8), pp.3640-3645, 2016.

[16] P. Paul, K. Kandasamy, M. Sharawi, A Tri-band Circularly Polarized Strip and SRR Loaded Slot Antenna, IEEE Transactions on Antennas and Propagation, 66 (10), pp.5569-5573, 2018.

[17] C. A. Balanis, Antenna Theory, Analysis and Design. 3rd ed, New York,NY, USA: Wiley, 2005.

[18] N. Engheta and R. W. Ziolkowski, Physics and Engineering Explorations, New York, NY, USA: Wiley, 2006.

[19] A. Ishikawa, T. Tanaka, and S. Kawata, Frequency Dependence of the magnetic response of split-ring resonators, J. Opt. Soc. Amer. B, 24 (3), pp. 510-515, 2007.

[20] D. Sarkar, K. Saurav, and K. V. Srivastava, Multi-band microstrip-fed slot antenna loaded with a split-ring resonator (SRR), Electron. Lett., 50 (21), pp. 1498-1500, 2014

[21] Esfandiari, Reza, Douglas W. Maki, and Mario Siracusa, Design of interdigitated capacitors and their application to gallium arsenide monolithic filters, IEEE Transactions on Microwave Theory and Techniques, 31(1), pp.57-64, 1983. 
[22] Withayachumnankul, Withawat, Christophe Fumeaux, and Derek Abbott, Compact electric-LC resonators for metamaterials, Optics Express, 18 (25), pp. 2591225921, 2010.

Table 1: Performance parameters of different multiband circularly polarized slot antennas $\left(\lambda_{\mathrm{g}}\right.$-guided wavelength corresponding to the lowest resonant frequency)

\begin{tabular}{|c|c|c|c|c|c|c|c|}
\hline REF. & SIZE & $\begin{array}{l}\text { RESONANCE } \\
\text { BANDS (GHz) }\end{array}$ & $\begin{array}{l}\text { IMPEDANCE } \\
\text { BW } \\
(\%)\end{array}$ & $\begin{array}{l}\text { ARBW } \\
(\%)\end{array}$ & $\begin{array}{l}\text { PEAK } \\
\text { GAIN } \\
(\text { dBi) }\end{array}$ & $\begin{array}{l}\text { RADIATION } \\
\text { EFFICIENCY } \\
(\%)\end{array}$ & $\begin{array}{l}\text { CONTROL OF } \\
\text { POLARIZATION } \\
\text { SENSE } \\
(\text { LHCP/RHCP) }\end{array}$ \\
\hline [7] & $0.6 \lambda_{g}^{2}$ & $\begin{array}{ll}\text { Two- } & 1.227, \\
1.575 & \end{array}$ & $3.7,1.2$ & $0.9,0.6$ & $1.1,1.45$ & $86.5,80.7$ & NO \\
\hline [5] & $1.74 \lambda_{g}^{2}$ & Two- $3.53,5.08$ & $33.16,22.72$ & $\begin{array}{l}1.7,3.86, \\
5.23\end{array}$ & $\begin{array}{l}5.5,4.63, \\
6.77\end{array}$ & ------------- & $\mathrm{NO}$ \\
\hline [6] & $1.58 \lambda_{g}^{2}$ & Two- $3,7.5$ & $44,70.9$ & $\begin{array}{l}35.9,44, \\
6.3\end{array}$ & $\begin{array}{ll}4.2, & 3.7, \\
3.5 & \end{array}$ & -------- & $\mathrm{NO}$ \\
\hline [8] & $0.5 \lambda_{g}^{2}$ & $\begin{array}{ll}\text { Three- } & 1.22, \\
1.57,2.31 & \end{array}$ & $9,2.5,27$ & $\begin{array}{ll}4.9, & 1.2, \\
4.3 & \end{array}$ & $4,4.6,5.7$ & ------- & NO \\
\hline [15] & $2.1 \lambda_{g}^{2}$ & Two- $3.1,4.7$ & $12.9,8.5$ & $3.1,4.2$ & $7.3,8.5$ & ----- & YES \\
\hline [16] & $0.3 \lambda_{g}^{2}$ & $\begin{array}{l}\text { Three- } 1.83,2.5 \text {, } \\
3.1\end{array}$ & $21.4,12.8,4.5$ & $\begin{array}{l}4.37, \\
11.9,3.6\end{array}$ & $\begin{array}{ll}2.7, & 4.2, \\
3.5 & \end{array}$ & $75,90,82$ & YES \\
\hline Proposed & $0.2 \lambda^{2} g$ & $\begin{array}{l}\text { Three- 1.5, 2.75, } \\
3.16\end{array}$ & $10,9.1,7.3$ & $\begin{array}{l}9.3,7.3, \\
8.5\end{array}$ & $\begin{array}{ll}3.2, & 4.5, \\
3.5 & \end{array}$ & $80,94,85$ & YES \\
\hline
\end{tabular}

